\title{
CONTROL AND ESTIMATION FOR A CLASS OF IMPULSIVE DYNAMICAL SYSTEMS
}

\author{
Tatiana F. Filippova \\ Krasovskii Institute of Mathematics and Mechanics, \\ Ural Branch of the Russian Academy of Sciences, \\ 16 S. Kovalevskaya Str., Ekaterinburg, 620990, Russia \\ Ural Federal University, \\ 19 Mira str., Ekaterinburg, 620002, Russia \\ ftf@imm.uran.ru
}

\begin{abstract}
The nonlinear dynamical control system with uncertainty in initial states and parameters is studied. It is assumed that the dynamic system has a special structure in which the system nonlinearity is due to the presence of quadratic forms in system velocities. The case of combined controls is studied here when both classical measurable control functions and the controls generated by vector measures are allowed. We present several theoretical schemes and the estimating algorithms allowing to find the upper bounds for reachable sets of the studied control system. The research develops the techniques of the ellipsoidal calculus and of the theory of evolution equations for set-valued states of dynamical systems having in their description the uncertainty of set-membership kind. Numerical results of system modeling based on the proposed methods are included.
\end{abstract}

Keywords: Control systems, Nonlinearity of quadratic type, Uncertainty, Impulse control, Ellipsoidal calculus, Tube of trajectories

\section{Introduction}

The paper is devoted to the state estimation problems for nonlinear control systems with uncertainty in description of their models. One of the central places in the theory of optimal control of dynamical systems is occupied by questions of constructing the corresponding reachable sets of the studied controlled systems, that is, the sets of all system positions obtained at a given time from a fixed initial state (or a set of such states) when all admissible controls are applied. Analysis of reachable sets and the construction of their different estimates may greatly facilitate the solution of many theoretical and applied problems of mathematical control theory. For linear controlled systems, the problem of describing and finding reachable sets has been considered in many papers and numerous ideas were involved to obtain external and internal estimates of reachable sets, basing on the corresponding versions of the ellipsoidal and polyhedral calculus [7, 8, 24, 26, 28, 35]. Note that even for linear systems studied at that time, the assumption that there are different kinds of uncertainties in describing the dynamics of systems significantly complicated the problem and transferred it to the class of nonlinear optimization problems.

A new stage in the development of approaches to solving nonlinear problems of estimating the states of control systems with uncertainty was carried out in connection with important researches in the field of set-valued analysis and in the theory of differential inclusions, including studies of sets of trajectories of control systems or differential inclusions with additional state constraints (the viability theory) [2, 27, 29, 32, 36, 37].

In this paper we study the case of a set-membership uncertainty [26-29, 32, 35] when only upper bounds on uncertain items are known and any additional probability characteristics for uncertainties are not done. Under such informational assumptions it is not possible to construct 
precisely related reachable sets of the dynamical control system but instead we may find external and (or) internal estimating sets for them using simple canonical structures (for example, ellipsoids or polyhedra). The proposed approaches are motivated by the development of the theory of uncertain control systems and can be used in further researches related to filtering, forecasting and smoothing problems for mechanical systems described by stochastic differential equations, multistep equations and inclusions, these results may help in solving a range of optimization problems for nonlinear controlled systems with impulse control, state constraints and uncertainty, they may be used also in the study of irregular problems of optimal control and in studies of resistance movements systems with generalized controls and with a delay uncertainty.

The approaches presented in this paper are based on main ideas of early research $[2,9,27]$ and are further developed for a different and more complicated classes of uncertain systems, the research continues and develops the results of the most recent studies [8, 11, 13, 15-19] for a wider class of control systems. Here we study the problems of constructing and estimating reachable sets of dynamical systems with impulse control $[10,12]$ and with uncertainty in the parameters of the systems dynamics and in the specification of its initial state. We further develop here the approaches related to consideration of bilinear uncertainties using the Minkowski gauge functionals [20].

Here we consider a more complicated case of a dynamic system than in papers [10, 12, 21], and we assume here that the impulse controls in the system are vectorial, which somewhat complicates both the previous analysis of the system dynamics and the corresponding proposed constructions, as well as the basic algorithm for constructing external estimates of reachable sets. Note that the issues of constructing internal ellipsoidal estimates of reachable sets of control systems with generalized (impulse) controls in both scalar and vector cases are much more complicated and are under development.

The results given here may be used in model-based advanced control of complex systems, such as adaptive control, robust control, sliding-mode control, H-infinite control, etc. [1, 3-6, 23, 25, 30]. Methods and schemes proposed in the paper possess such features as reliability, sufficient simplicity of computational algorithms and relatively high speed of their processing, so these schemes allow using them in real time e.g. in problems of robust control, stability, problems of control synthesis for dynamic systems of various types including problems of forecasting financial results in economic planning and other fields.

The paper is organized as follows. We introduce first some notations and definitions and formulate the main problem in Section 2. The approach related to upper estimates of reachable sets in nonlinear case under study is described in Section 3. Example illustrating the results is given in Section 4. Finally, some concluding remarks are given.

\section{Problem formulation}

In this section we introduce some basic notations and constructions and formulate further the main problem of state estimation for nonlinear control system with uncertainty and with impulsive controls of vector type.

\subsection{Main notations}

Let $\mathbb{R}^{n}$ denote the $n$-dimensional Euclidean space and $x^{\prime} y$ is the usual inner product of vectors $x, y \in \mathbb{R}^{n}$ (the prime corresponds to a transpose), $\|x\|=\left(x^{\prime} x\right)^{1 / 2}$. We will use also other norms of $x=\left(x_{1}, \ldots, x_{n}\right) \in \mathbb{R}^{n}$, namely $\|x\|_{p}=\left(\sum_{i=1}^{n}\left|x_{i}\right|^{p}\right)^{1 / p}$ for $1 \leq p<\infty$. The symbol comp $\mathbb{R}^{n}$ stands for the variety of all compact subsets $A \subset \mathbb{R}^{n}$ and conv $\mathbb{R}^{n}$ corresponds to a variety of all compact convex subsets $A \subset \mathbb{R}^{n}$. 
Denote by clconv $\mathbb{R}^{n}$ the set of all closed convex subsets $A \subseteq \mathbb{R}^{n}$. Let $\mathbb{R}^{n \times m}$ be the set of all $n \times m$-matrices, $\operatorname{diag}\{v\}$ denotes a diagonal matrix with elements of a vector $v$ standing at the main diagonal (and with zeros at other places). Let $I \in \mathbb{R}^{n \times n}$ be the identity matrix and $\operatorname{Tr}(A)$ be the trace of $n \times n$-matrix $A=\left\{a_{i j}\right\}$ (the sum of its diagonal elements, $\left.\operatorname{Tr}(A)=\sum_{i=1}^{n} a_{i i}\right)$.

We denote also as $B(a, r)=\left\{x \in \mathbb{R}^{n}:\|x-a\| \leq r\right\}$ the ball in $\mathbb{R}^{n}$ with a center $a \in \mathbb{R}^{n}$ and a radius $r>0$ and denote as

$$
E(a, Q)=\left\{x \in \mathbb{R}^{n}:\left(Q^{-1}(x-a),(x-a)\right) \leq 1\right\}
$$

the ellipsoid in $\mathbb{R}^{n}$ with a center $a \in \mathbb{R}^{n}$ and with a symmetric positive definite $n \times n$-matrix $Q$.

\subsection{Problem description}

Consider the following impulsive control system $\left(t_{0} \leq t \leq T, x \in \mathbb{R}^{n}\right)$

$$
\begin{aligned}
d x(t)= & \left(A(t) x(t)+x^{\prime} B x \cdot d+u(t)\right) d t+C d v(t), \\
& x\left(t_{0}-0\right)=x_{0} \in X_{0}=E\left(a_{0}, Q_{0}\right) .
\end{aligned}
$$

Here a matrix $A(t)$ is unknown but satisfies the constraint

$$
A(t) \in \mathcal{A}=A^{0}+\mathcal{A}^{1}, \quad t_{0} \leq t \leq T,
$$

where $A^{0}$ is a given matrix and

$$
\begin{gathered}
\mathcal{A}^{1}=\left\{A=\left\{a_{i j}\right\} \in \mathbb{R}^{n \times n}: a_{i j}=0 \text { for } i \neq j, \quad\right. \text { and } \\
\left.a_{i i}=a_{i}, \quad i=1, \ldots, n, \quad a=\left(a_{1}, \ldots, a_{n}\right), \quad a^{\prime} D a \leq 1\right\},
\end{gathered}
$$

with $D \in \mathbb{R}^{n \times n}$ being a given symmetric and positive definite matrix.

We assume that the impulsive part $v:\left[t_{0}, T\right] \rightarrow \mathbb{R}^{m}$ of the control pair $\{u(\cdot), v(\cdot)\}$ in (1.1) is of bounded variation on $\left[t_{0}, T\right]$, with

$$
\operatorname{Var}_{t \in\left[t_{0}, T\right]} v(t)=\sup _{\left\{t_{i}\right\}}\left\{\sum_{i=1}^{k}\left\|v\left(t_{i}\right)-v\left(t_{i-1}\right)\right\|_{1}: \quad \forall t_{i}: t_{0} \leq t_{1} \leq \ldots \leq t_{k}=T\right\} \leq \mu,
$$

where $\mu>0$ is given. Denote the above class of functions $v(\cdot)$ as $\mathcal{V}$.

We assume also that $u(t) \in \mathcal{U}=E(\hat{a}, \hat{Q})$ where the center $\hat{a}$ and the matrix $\hat{Q}$ of the ellipsoid $\mathcal{U}$ are known.

The guaranteed estimation problem consists in describing the set

$$
\begin{gathered}
\mathcal{X}(t)=\mathcal{X}\left(t ; t_{0}, X_{0}\right)=\left\{x \in \mathbb{R}^{n}: \exists x_{0} \in X_{0}, \exists u(\cdot) \in \mathcal{U}, \exists v(\cdot) \in \mathcal{V}, \exists A(\cdot) \in \mathcal{A}^{1}\right. \\
\text { such that } \left.x=x(t)=x\left(t ; u(\cdot), v(\cdot), x_{0}, A(\cdot)\right)\right\} .
\end{gathered}
$$

of solutions to the system (1.1)-(1.2).

The problem studied here is to construct external ellipsoidal estimates for reachable sets $\mathcal{X}(t)$ $\left(t_{0}<t \leq T\right)$ basing on recent results and on related techniques of the estimation theory for control systems with uncertainty and nonlinearity. We investigate a more complicated case than in $[15,17]$ and use here the technique recently developed in [19]. The main ideas used to solve the estimation problem go back to the results and reparametrization procedure of the papers [10, 31], with corresponding changes and improvements caused by the presence of vector measures (generalized controls). 


\section{Problem solution}

The main result of the paper is connected with a special scheme of transition from a system of impulse type to a control system (or the corresponding differential inclusion) that does not contain impulse control components. Note that the proposed construction differs from the schemes of $[10,12,21]$ where the case of scalar impulse components of control components was investigated.

\subsection{Auxiliary constructions: impulsive differential inclusions}

Consider a differential inclusion of the following type

$$
d x(t) \in F(t, x(t)) d t+C(t) d v(t),
$$

with the initial condition

$$
x\left(t_{0}-0\right)=x_{0}, \quad x_{0} \in X_{0} .
$$

Here we use the notation

$$
F(t, x)=f(t, x, U)=\bigcup\{f(t, x, u): u \in U\} .
$$

Definition 1 [33]. A function $x[t]=x\left(t, t_{0}, x_{0}\right) \quad\left(x_{0} \in X_{0}, t \in\left[t_{0}, T\right]\right)$ will be called a solution (a trajectory) of the differential inclusion (2.1) if for all $t \in\left[t_{0}, T\right]$ the following equality holds true

$$
x[t]=x_{0}+\int_{t_{0}}^{t} \psi(t) d t+\int_{t_{0}}^{t} C(t) d v(t),
$$

where $\psi(\cdot) \in L_{1}^{n}\left[t_{0}, T\right]$ is a selector of $F$, that is $\psi(t) \in F(t, x[t])$ a.e. (the last integral in (2.2) is taken as the Riemann-Stieltjes integral).

Following the scheme of the proof of the well-known Caratheodory theorem we can prove the existence of solutions $x(\cdot)=x\left(\cdot, t_{0}, x_{0}\right) \in B V^{n}\left[t_{0}, T\right]$ for all $x_{0} \in X_{0}$ where $B V^{n}\left[t_{0}, T\right]$ is the space of $n$-vector functions with bounded variation at $\left[t_{0}, T\right]$.

\subsection{Discontinuous time replacement}

Let us introduce a new time variable [10,31, 34],

$$
\eta(t)=t+\int_{t_{0}}^{t}\|d v(t)\|_{1},
$$

and a new state coordinate $\tau(\eta)=\inf \{t: \eta(t) \geq \eta\}$. Consider the following auxiliary differential inclusion

$$
\frac{d}{d \eta}\left(\begin{array}{c}
z \\
\tau
\end{array}\right) \in H(\tau, z)
$$

with the initial condition

$$
z\left(t_{0}\right)=x^{0}, \quad \tau\left(t_{0}\right)=t_{0}, \quad t_{0} \leq \eta \leq T+\mu .
$$

Here we denote

$$
H(\tau, z)=\bigcup_{0 \leq \nu \leq 1}\left\{\nu\left(\begin{array}{c}
C^{*} \\
0
\end{array}\right)+(1-\nu)\left(\begin{array}{c}
A z+z^{\prime} B z \cdot d+E(\hat{a}, \hat{Q}) \\
1
\end{array}\right)\right\},
$$


where $C^{*}=\operatorname{co}\left\{c^{(1)}, \ldots, c^{(m)}\right\}$ and $c^{(i)} \in \mathbb{R}^{n}(i=1, \ldots, m)$ are columns of the matrix $C \in \mathbb{R}^{n \times m}$.

Under the above assumptions on the impulsive system we have two lemmas which will be used in further analysis.

Lemma 1. The map $H(\tau, z)$ is convex and compact valued

$$
H:\left[t_{0}, T+\mu\right] \times \mathbb{R}^{n} \rightarrow \operatorname{conv} \mathbb{R}^{n+1}
$$

and $H(\tau, z)$ is Lipschitz continuous in both variables $\tau, z$.

P r o o f. The required properties can be easily derived from the specific type of set-valued map $H(\tau, z)$ defined above.

Remark 1. Note that the design of the auxiliary differential inclusion (2.3) is different from the scheme used in [14]. The reason is the assumption of a vector type for impulse controls in (2.3)-(2.4). We also indicate that in the paper [14] a different type of constraints on undefined elements of the matrix $\mathcal{A}^{1}$ (in (1.2)) was investigated.

Denote $w=\{z, \tau\}$ the extended state vector of the system (2.3) and consider trajectory tube of this differential inclusion (which has no measure or impulse components):

$$
W(\eta)=\bigcup_{w^{0} \in X^{0} \times\left\{t_{0}\right\}} w\left(\eta, t_{0}, w^{0}\right), \quad t_{0} \leq \eta \leq T+\mu .
$$

The next lemma explains the construction of the auxiliary differential inclusion (2.3)-(2.4).

Lemma 2. The set $\mathcal{X}(T)$ is the projection of $W(T+\mu)$ at the subspace of state variables $z$ :

$$
\mathcal{X}(T)=\pi_{z} W(T+\mu) .
$$

P r o o f. The proof of this result can be carried out according to the scheme of the paper [10], with a slight modification due to a more complicated case of the vector measure $d v(t)$ in $(1.1)$ considered here.

Denote as $h_{M}(z)$ the Minkowski (gauge) functional for a set $M \subset \mathbb{R}^{n}[9,20]$,

$$
h_{M}(z)=\inf \left\{t>0: z \in t M, x \in \mathbb{R}^{n}\right\},
$$

and let $W\left(t ; t_{0}, X_{0} \times\left\{t_{0}\right\}\right)$ be a trajectory tube of the inclusion (2.3)-(2.4).

Denote as $E(\tilde{c}, \tilde{Q})$ the ellipsoid with minimal volume and such that

$$
C^{*} \subseteq E(\tilde{c}, \tilde{Q}) .
$$

Theorem 1. For any $\sigma>0$ the following inclusion is true

$$
W\left(t_{0}+\sigma\right) \subseteq \mathcal{W}\left(t_{0}, \sigma, \nu\right)+o(\sigma) B_{*}(0,1), \quad \lim _{\sigma \rightarrow+0} \sigma^{-1} o(\sigma)=0
$$

where

$$
\begin{gathered}
\mathcal{W}\left(t_{0}, \sigma, \nu\right)=\left(\begin{array}{c}
E\left(a^{*}(\sigma, \nu), Q^{*}(\sigma, \nu)\right) \\
t_{0}+\sigma(1-\nu)
\end{array}\right), \\
a^{*}(\sigma, \nu)=a_{0}+\sigma\left((1-\nu)\left(a_{0}{ }^{\prime} B a_{0} \cdot d+k^{2} d+\hat{a}\right)+\nu \tilde{c}\right), \\
Q^{*}(\sigma, \nu)=\left(p^{-1}+1\right) \tilde{Q}(\sigma, \nu)+(p+1) \sigma^{2} \hat{Q}_{\nu}^{*},
\end{gathered}
$$


with $E\left(\hat{a}_{\nu}, \hat{Q}_{\nu}^{*}\right)$ being the ellipsoid with minimal volume such that

$$
\begin{gathered}
\nu E(\tilde{c}, \tilde{Q})+(1-\nu) E(\hat{a}, \hat{Q})+2(1-\nu) d \cdot a_{0}^{\prime} B \cdot E\left(0, k^{2} B^{-1}\right) \subseteq E\left(\hat{a}_{\nu}, \hat{Q}_{\nu}^{*}\right), \\
\hat{a}_{\nu}=\nu \tilde{c}+(1-\nu) \hat{a},
\end{gathered}
$$

and where the function $\tilde{Q}(\sigma, \nu)$ in (2.6) is defined as follows,

$$
\tilde{Q}(\sigma, \nu)=\operatorname{diag}\left\{\left(p^{-1}+1\right) \sigma^{2} a_{0 i}^{2}+(p+1) r^{2}(\sigma): i=1, \ldots, n\right\},
$$

with

$$
r(\sigma)=\max _{z}\|z\| \cdot\left(h_{(I+\sigma \mathcal{A}) * \mathcal{X}_{0}}(z, \sigma)\right)^{-1},
$$

and $p=p(\sigma, \nu)$ is the unique positive root of the equation

$$
\sum_{i=1}^{n} \frac{1}{p+\lambda_{i}}=\frac{n}{p(p+1)}
$$

with numbers $\lambda_{i}=\lambda_{i}(\sigma, \nu) \geq 0(i=1, \ldots, n)$ satisfying the equation $\left|\tilde{Q}(\sigma, \nu)-\lambda \sigma^{2} \hat{Q}_{\nu}^{*}\right|=0$.

P r o o f. In order to calculate the upper estimate for $W\left[t_{0}+\sigma\right]$ we use first the inclusion (2.5) and therefore we may weaken the estimate (2.3)-(2.4) in the following way, considering the modified differential inclusion

$$
\frac{d}{d \eta}\left(\begin{array}{c}
z \\
\tau
\end{array}\right) \in H^{*}(\tau, z)
$$

with the initial condition

$$
z\left(t_{0}\right)=x^{0}, \quad \tau\left(t_{0}\right)=t_{0}, \quad t_{0} \leq \eta \leq T+\mu,
$$

where the set-valued map $H^{*}(\tau, z)$ is defined as

$$
H^{*}(\tau, z)=\bigcup_{0 \leq \nu \leq 1}\left\{\nu\left(\begin{array}{c}
E(\tilde{c}, \tilde{C}) \\
0
\end{array}\right)+(1-\nu)\left(\begin{array}{c}
A z+z^{\prime} B z \cdot d+E(\hat{a}, \hat{Q}) \\
1
\end{array}\right)\right\} .
$$

Estimating the sum of two ellipsoids $\nu E(\tilde{c}, \tilde{C})$ and $(1-\nu) E(\hat{a}, \hat{Q})$ in the above formula (see, e.g., related procedures in [7, 28]) and using the results of Theorem 3 in [22] we come to the relations $(2.6)-(2.8)$.

Remark 2. To determinate a better estimate of the reachable set $\mathcal{W}\left(t_{0}+\sigma\right)$ we may introduce a small parameter $\varepsilon>0$ and embed the set $\mathcal{W}\left(t_{0}, \sigma, \nu\right)$ into a nondegenerate ellipsoid $E_{\varepsilon}\left(w\left(t_{0}, \sigma, \nu\right), O_{\varepsilon}\left(t_{0}, \sigma, \nu\right)\right)$ :

$$
\begin{gathered}
\mathcal{W}\left(t_{0}, \sigma, \nu\right) \subseteq E_{\varepsilon}\left(w\left(t_{0}, \sigma, \nu\right), O_{\varepsilon}\left(t_{0}, \sigma, \nu\right)\right) \\
w\left(t_{0}, \sigma, \nu\right)=\left(\begin{array}{c}
a^{*}(\sigma, \nu) \\
t_{0}+\sigma(1-\nu)
\end{array}\right), \quad O_{\varepsilon}\left(t_{0}, \sigma, \nu\right)=\left(\begin{array}{cc}
Q^{*}(\sigma, \nu) & 0 \\
0 & \varepsilon^{2}
\end{array}\right) .
\end{gathered}
$$

For small $\varepsilon>0$ we will have

$$
\begin{aligned}
\mathcal{W}\left(t_{0}, \sigma\right) \subset \mathcal{W}_{\varepsilon}\left(t_{0}, \sigma\right)= & \bigcup_{0 \leq \nu \leq 1} E_{\varepsilon}\left(w\left(t_{0}, \sigma, \nu\right), O_{\varepsilon}\left(t_{0}, \sigma, \nu\right)\right) \subset E_{\varepsilon}\left(w^{+}(\sigma), O^{+}(\sigma)\right), \\
& \lim _{\varepsilon \rightarrow+0} h\left(\mathcal{W}\left(t_{0}, \sigma\right), \mathcal{W}_{\varepsilon}\left(t_{0}, \sigma\right)\right)=0,
\end{aligned}
$$


here $h(A, B)$ is the Hausdorff distance between compact sets $A, B \subset \mathbb{R}^{n}$.

Further as the next step of the describing estimation procedure we may use the algorithms developed in [21] and applying them we construct the upper estimate $E_{\varepsilon}\left(w^{+}(\sigma), O^{+}(\sigma)\right)$ for the union of ellipsoids $\mathcal{W}_{\varepsilon}\left(t_{0}, \sigma\right)$. Thus we get the ellipsoidal estimate of the reachable set $\mathcal{W}\left(t_{0}+\sigma\right)$

$$
\mathcal{W}\left(t_{0}+\sigma\right) \subset E_{\varepsilon}\left(w^{+}(\sigma), O^{+}(\sigma)\right)+o(\sigma) B(0,1) .
$$

Now we can formulate a new computational algorithm for the numerical construction of external ellipsoidal estimates for reachable sets of the system (1.1), this algorithm essentially uses the Theorem 1.

\section{Algorithm (External Estimation of Reachable Sets).}

Subdivide the time segment $\left[t_{0}, T+\mu\right]$ into subsegments $\left\{\left[t_{i}, t_{i+1}\right]\right\}$, where $t_{i}=t_{0}+i h$ $(i=1, \ldots, m), h=\left(T+\mu-t_{0}\right) / m, t_{m}=T+\mu$. Subdivide also the segment $[0,1]$ into subsegments $\left[\nu_{j}, \nu_{j+1}\right]$, where $\nu_{i}=i h_{*}, h_{*}=1 / m, \nu_{0}=0, \nu_{m}=1$.

1. Repeated steps begin with Step 1:

- Take $\sigma=h$ and for given $X_{0}=E\left(a_{0}, k^{2} B^{-1}\right)$ define by Theorem 1 the sets $\mathcal{W}\left(t_{0}, \sigma, \nu_{i}\right)$ $(i=0, \ldots, m)$.

- Find ellipsoid $E_{\varepsilon}\left(w_{1}(\sigma), O_{1}(\sigma)\right)$ in $\mathbb{R}^{n+1}$ such that

$$
\mathcal{W}\left(t_{0}, \sigma, \nu_{i}\right) \subseteq E_{\varepsilon}\left(w_{1}(\sigma), O_{1}(\sigma)\right) \quad(i=0, \ldots, m) .
$$

At this step we find the ellipsoidal estimate for the union of a finite family of ellipsoids [21].

- Find the projection $E\left(a_{1}, Q_{1}\right)=\pi_{z} E_{\varepsilon}\left(w_{1}(\sigma), O_{1}(\sigma)\right)$ by Lemma 2 .

- Find the smallest $k_{1}>0$ such that $E\left(a_{1}, Q_{1}\right) \subseteq E\left(a_{1}, k_{1}^{2} B^{-1}\right)\left(k_{1}^{2}\right.$ is the maximal eigenvalue of the matrix $B^{1 / 2} Q_{1} B^{1 / 2}$ ).

- Consider the system on the next subsegment $\left[t_{1}, t_{2}\right]$ with $E\left(a_{1}, k_{1}^{2} B^{-1}\right)$ as the initial ellipsoid at instant $t_{1}$.

2. The next step repeats the previous iteration beginning with new initial data. At the end of the process we will get the external estimate $E\left(a^{+}(T), Q^{+}(T)\right)$ of the reachable set of the system (1.1)-(1.3).

Remark 3. One of the subsequent steps of the above algorithm contains the projection of an ellipsoid on the subspace of the part of state variables, it complicates a bit the whole estimation procedure. But it is not possible to avoid this difficult step of the whole estimation process because of the presence of impulsive components in the control functions. One of the main goals of this paper is to overcome this complication.

\section{Example}

In this section we illustrate the main ideas and results obtained above by an example of an impulsive control system with uncertain initial set and with nonlinearity in dynamics. 
Example. Consider the following control system

$$
\left\{\begin{array}{l}
d x_{1}=a_{1} x_{1} d t+u_{1}(t) d t+d v_{1} \\
d x_{2}=a_{2} x_{2} d t+x_{1}^{2} d t+x_{2}^{2} d t+u_{2}(t) d t+0.01 d v_{2},
\end{array} \quad 0 \leq t \leq T,\right.
$$

with unknown initial state which belongs to a unit ball

$$
x_{0} \in X_{0}=B(0,1) .
$$

Here we take $t_{0}=0, T=0.4, \mathcal{U}=B(0, r)$, with $r=0.01$. We have also $A=2 I, B=I, d_{1}=0$, $d_{2}=1$.

External ellipsoidal tube $E^{+}(t)=E\left(a^{+}(t), Q^{+}(t)\right)$ is shown at Fig. 1, it is found using the main result of Theorem 1 and is constructed according to the the main Algorithm. The first estimating ellipsoid $E\left(0, k_{0}^{2} B^{-1}\right)$ is shown in red color and it contains $X_{0}$ (it is shown in blue color). It is worth recalling that the construction of the set $E\left(0, k_{0}^{2} B^{-1}\right)$ begins the whole iterative estimation process described by the Algorithm.

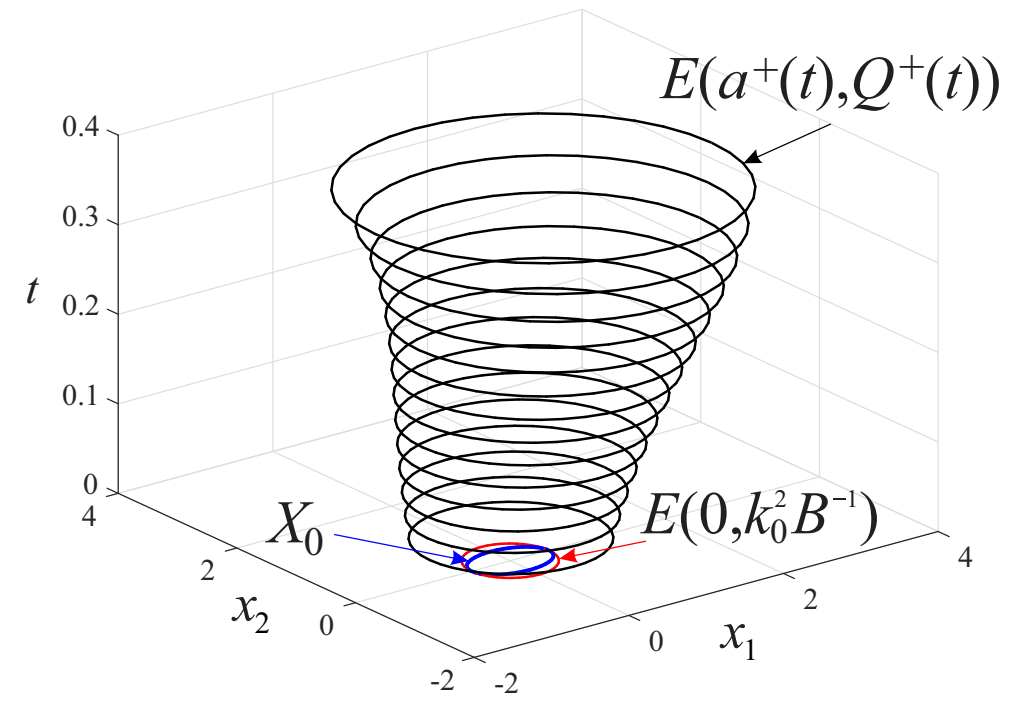

Figure 1. External ellipsoidal tube $E^{+}(t)=E\left(a^{+}(t), Q^{+}(t)\right), t \in[0,0.4]$.

Remark 4. The example shows that the estimation errors can increase with time (accumulation effect). However, this is due to two factors of the model, the presence of nonlinear terms in the equations of dynamics and the presence of impulse controls.

\section{Conclusions}

The problems of state estimation for nonlinear impulsive control systems with unknown but bounded initial states were studied here. The solution was implemented based on the techniques of trajectory tubes of differential inclusions theory and also based on results of ellipsoidal calculus developed recently for these class of problems.

We study here the case when the system nonlinearity is generated by the combination of two types of functions in related differential equations, one of which is bilinear and the other one is quadratic. Additional difficulties in solving the considered problems were caused by the presence in the dynamic system of impulsive actions of a vector type. 
The applications of the problems studied in this paper are in guaranteed state estimation for nonlinear systems with unknown but bounded errors and in related applied fields (e.g., in robotics, in problems of motor actuation, hydraulic actuation and others fields), the approaches developed here may be used in the model-based advanced control of complex systems, such as adaptive control, robust control, sliding-mode control, H-infinite control, etc.

Directions for further investigation continuing the paper research may be motivated by the studies in the theory of dynamic systems with uncertainty and with vector impulse controls under more complicated assumptions e.g. when the right hand sides of differential equations describing the system dynamics contain the product of state coordinates and the generalized (impulse) controls.

\section{REFERENCES}

1. Asselborn L., Groß D., Stursberg O. Control of uncertain nonlinear systems using ellipsoidal reachability calculus. IFAC Proc. Volumes, 2013. Vol. 46, no. 23. P. 50-55. DOI: 10.3182/20130904-3-FR-2041.00204

2. Aubin J.-P., Frankowska H. Set-Valued Analysis. Basel: Birkhäuser, 1990. 461 p.

3. August E., Lu J., Koeppl H. Trajectory enclosures for nonlinear systems with uncertain initial conditions and parameters. In: Proc. of the 2012 American Control Conf., June 27-29, 2012, Montréal, Canada. QC. IEEE Computer Soc., 2012. P. 1488-1493.

4. Blanchini F., Miani S. Set-Theoretic Methods in Control. Ser. Syst. Control: Foundations \& Applications. Birkhäuser, Basel, 2015. XV+487 p. DOI: 10.1007/978-0-8176-4606-6

5. Boscain U., Chambrion T., Sigalotti M. On some open questions in bilinear quantum control. In: Proc. of the European Control Conf. (ECC), July 17-19, 2013, Zurich, Switzerland. IEEE Xplore, 2013. P. 2080-2085. DOI: 10.23919/ECC.2013.6669238

6. Ceccarelli N., Di Marco M., Garulli A., Giannitrapani A. A set theoretic approach to path planning for mobile robots. In: Proc. 43rd IEEE Conf. on Decision and Control (CDC) Dec. 14-17, 2004, Nassau, Bahamas. IEEE Xplore, 2004. P. 147-152. DOI: 10.1109/CDC.2004.1428621

7. Chernousko F. L. State Estimation for Dynamic Systems. CRC Press: Boca Raton, 1994. 320 p.

8. Chernousko F.L., Rokityanskii D. Ya. Ellipsoidal bounds on reachable sets of dynamical systems with matrices subjected to uncertain perturbation. J. Optim. Theory Appl., 2000. Vol. 104, No. 1. P. 1-19. DOI: $10.1023 / \mathrm{A}: 1004687620019$

9. Demyanov V.F., Rubinov A.M. Quasidifferential Calculus. New York: Optimization Software Inc., 1986.

10. Filippova T.F. Set-valued solutions to impulsive differential inclusions. Math. Comput. Model. Dyn. Syst., 2005. No. 11. P. 149-158.

11. Filippova T.F. Differential equations of ellipsoidal state estimates in nonlinear control problems under uncertainty. Discrete Contin. Dyn. Syst., Vol. Suppl.-2011. 2011 P. 410-419. DOI: 10.3934 /proc.2011.2011.410

12. Filippova T.F. Approximation techniques in impulsive control problems for the tubes of solutions of uncertain differential systems. Advances in Applied Mathematics and Approximation Theory. Springer Proc. Math. Stat., vol. 41. New York: Springer, 2013. P. 385-396. DOI: 10.1007/978-1-4614-6393-125

13. Filippova T.F. State estimation for a class of nonlinear dynamic systems with uncertainty through dynamic programming technique. In: Proc. of the 6th Int. Conf. PhysCon2013, August 26-29, 2013, San Lois Potosi, Mexico, 2013. P. 1-6.

14. Filippova T.F. Estimating reachable sets of control systems with uncertainty on initial data and with nonlinearity of a special kind. In: Proc. of the Int. Conf. Stability and Oscillations of Nonlinear Control Systems (Pyatnitskiy's Conference), June 1-3, 2016, Moscow, Russia. IEEE Xplore, 2016. P. 1-4. DOI: $10.1109 /$ STAB.2016.7541183

15. Filippova T.F. Ellipsoidal estimates of reachable sets for control systems with nonlinear terms. IFACPapersOnLine, 2017. Vol. 50, No. 1. P. 15355-15360. DOI: 10.1016/j.ifacol.2017.08.2460

16. Filippova T.F. Dynamics and estimates of star-shaped reachable sets of nonlinear control systems. J. Chaotic Modeling and Simulation (CMSIM), 2017. No. 4. P. 469-478.

17. Filippova T.F. Estimation of star-shaped reachable sets of nonlinear control system. In: Lecture Notes in Comput. Sci., vol. 10665: Proc. Large-Scale Scientific Computing. LSSC 201\%. Cham: Springer, 2018. P. 210-218. DOI: 10.1007/978-3-319-73441-5_22 
18. Filippova T.F. Differential equations for ellipsoidal estimates of reachable sets for a class of control systems with nonlinearity and uncertainty. IFAC-PapersOnLine, 2018. Vol. 51, No. 32. P. 770-775. DOI: $10.1016 /$ j.ifacol.2018.11.452

19. Filippova T.F. Description of dynamics of ellipsoidal estimates of reachable sets of nonlinear control systems with bilinear uncertainty. In: Lecture Notes in Comput. Sci., vol. 11189: Numerical Methods and Applications. NMA 2018. Cham: Springer, 2019. P. 97-105. DOI: 10.1007/978-3-030-10692-8_11

20. Filippova T. F., Lisin D. V. On the estimation of trajectory tubes of differential inclusions. Proc. Steklov Inst. Math., 2000. Vol. Suppl. 2. P. S28-S37.

21. Filippova T.F., Matviychuk O.G. Algorithms to estimate the reachability sets of the pulse controlled systems with ellipsoidal phase constraints. Autom. Remote Control, 2011. Vol. 72, No. 9. P. 1911-1924. DOI: 10.1134/S000511791109013X

22. Filippova T.F., Matviychuk O. G. Algorithms of estimating reachable sets of nonlinear control systems with uncertainty. J. Chaotic Modeling and Simulation, 2015. No. 3. P. 205-214.

23. Kishida M., Braatz R. D. Ellipsoidal bounds on state trajectories for discrete-time systems with linear fractional uncertainties. Optim. Eng., 2015. Vol. 16. P. 695-711. DOI: 10.1007/s11081-014-9255-9

24. Kostousova E.K., Kurzhanski A. B. Theoretical framework and approximation techniques for parallel computation in set-membership state estimation. In: Proc. of the Symposium on Modelling Analysis and Simulation, July 9-12, 1996, Lille, France, 1996. No. 2. P. 849-854.

25. Kuntsevich V. M., Volosov V. V. Ellipsoidal and interval estimation of state vectors for families of linear and nonlinear discrete-time dynamic systems. Cybernet. Systems Anal., 2015. Vol. 51. P. 64-73. DOI: $10.1007 /$ s10559-015-9698-9

26. Kurzhanski A. B. Upravlenie i nablyudenie v usloviyah neopredelennosti [Control and Observation under Conditions of Uncertainty]. Moscow: Nauka, 1977. 392 p. (in Russian)

27. Kurzhanski A.B., Filippova T.F. On the theory of trajectory tubes - a mathematical formalism for uncertain dynamics, viability and control. In: Advances in Nonlinear Dynamics and Control: a Report from Russia, ed. A. B. Kurzhanski. Progress in Systems and Control Theory, vol. 17. Boston: Birkhäuser, 1993. P. 122-188. DOI: 10.1007/978-1-4612-0349-0_4

28. Kurzhanski A.B., Valyi I. Ellipsoidal Calculus for Estimation and Control. Systems Control Found. Appl. Basel: Birkhäuser, 1997. 321 p.

29. Kurzhanski A. B., Varaiya P. Dynamics and Control of Trajectory Tubes: Theory and Computation. Systems Control Found. Appl., vol. 85. Basel: Birkhäuser, 2014. 445 p. DOI: 10.1007/978-3-319-10277-1

30. Malyshev V.V., Tychinskii Yu. D. Construction of sets of attainability and maneuver optimization for low-thrust artificial satellites of the earth in a strong gravitational field. J. Comput. Syst. Sci. Int., 2005. Vol. 44, No. 4. P. 622-630.

31. Miller B. M. Method of discontinuous time change in problems of control for impulse and discretecontinuous systems. Autom. Remote Control, 1993. Vol. 54, No. 12. P. 1727-1750.

32. Panasyuk A. I. Equations of attainable set dynamics. Part 1: Integral funnel equations. J. Optimiz. Theory Appl., 1990. Vol. 64. P. 349-366. DOI: 10.1007/BF00939453

33. Pereira F. L., Filippova T.F. On a solution concept to impulsive differential systems. In: Proc. of 4th Int. Conf. Tools for Mathematical Modelling (MathTools'03), June 23-28, 2003, St.Petersburg, Russia. 2003. P. 350-355.

34. Rishel R. An extended Pontryagin principle for control system whose control laws contain measures. SIAM J. Control, 1965. Vol. 3. P. 191-205.

35. Schweppe F. C. Uncertain Dynamic Systems. New Jersey: Prentice-Hall, Englewood Cliffs, 1973.563 p.

36. Veliov V.M. Second order discrete approximations to strongly convex differential inclusions. Systems Control Lett., 1989. Vol. 13. P. 263-269.

37. Veliov V. M. Second-order discrete approximation to linear differential inclusions. SIAM J. Numer. Anal., 1992. Vol. 29, no. 2. P. 439-451. 